\title{
Pleiotropic effects of polymorphism of the gene diacylglycerol- $O$ - transferase 1 (DGAT1) in the mammary gland tissue of dairy cows
}

\author{
N. Mach, ${ }^{11,2}$ Y. Blum, $† \ddagger$ A. Bannink, ${ }^{*}$ D. Causeur, $\neq$ M. Houee-Bigot, $\neq$ S. Lagarrigue, $†$ and M. A. Smits* \\ *Wageningen UR Livestock Research, Lelystad 8200 AB, the Netherlands \\ †Agrocampus Ouest, UMR598, Génétique Animale, Rennes 35000, France \\ $\ddagger$ Agrocampus Ouest, Applied Mathematics, Rennes 35000, France
}

\section{ABSTRACT}

Microarray analysis was used to identify genes whose expression in the mammary gland of Holstein-Friesian dairy cows was affected by the nonconservative Ala to Lys amino acid substitution at position 232 in exon VIII of the diacylglycerol- $O$-transferase 1 (DGAT1) gene. Mammary gland biopsies of 9 homozygous Ala cows, 13 heterozygous cows (Ala/Lys), and 4 homozygous Lys cows in midlactation were taken. Microarray ANOVA and factor analysis for multiple testing methods were used as statistical methods to associate the expression level of the genes present on Affymetrix bovine genome arrays (Affymetrix Inc., Santa Clara, CA) with the DGAT1 gene polymorphism. The data was also analyzed at the level of functional modules by gene set enrichment analysis. In this small-scale experimental setting, DGAT1 gene polymorphism did not modify milk yield and composition significantly, although expected changes occurred in the yields of C14:0, cis-9 C16:1, and long-chain fatty acids. Diacylglycerol- $O$-transferase 1 gene polymorphism affected the expression of 30 annotated genes related to cell growth, proliferation, and development, remodeling of the tissue, cell signaling and immune system response. Furthermore, the main affected functional modules were related to energy metabolism (lipid biosynthesis, oxidative phosphorylation, electron transport chain, citrate cycle, and propanoate metabolism), protein degradation (proteosome-ubiquitin pathways), and the immune system. We hypothesize that the observed differences in transcriptional activity reflect counter mechanisms of mammary gland tissue to respond to changes in milk fatty acid concentration or composition, or both.

Key words: diacylglycerol-O-transferase 1 (DGAT1) gene, lactating cow, mammary gland, microarray

Received January 16, 2012.

Accepted May 4, 2012.

${ }^{1}$ Corresponding author: nuria.mach@jouy.inra.fr

${ }^{2}$ This author is currently working at INRA UMR 1313 Génétique Animale et Biologie Intégrative, 78352 Jouy-en-Josas, France.

\section{INTRODUCTION}

The diacylglycerol- $O$-transferase 1 (DGAT1) gene is mapped to the centromeric end of bovine chromosome 14 (Boichard et al., 2003) and encodes the DGAT1 microsomal enzyme, which catalyzes the last step in triglyceride (TAG) synthesis: the esterification of a fatty acyl-CoA to the $s n-3$ position of a diacylglycerol. Evidence exists that the nonconservative Ala (A) to Lys $(\mathbf{K})$ amino acid substitution at position 232 in exon VIII of the DGAT1 gene is associated with decreased milk production (Schennink et al., 2007; Banos et al., 2008; Berry et al., 2010), decreased milk protein yield (Thaller et al., 2003; Schennink et al., 2007), increased milk fat yield (Thaller et al., 2003; Schennink et al., 2007), and changes in milk FA composition (Schennink et al., 2007, 2008; Bouwman et al., 2011). The DGAT1 $232 \mathrm{~K}$ allele is associated with increased saturated fat and reduced fractions of unsaturated C18 FA and conjugated linoleic acid in milk, which is regarded as unfavorable to human health. Therefore, from the human health perspective, decreasing the frequency of the DGAT1 232K allele in dairy cows through breeding programs could be desirable because milk and milkderived foods are still large contributors to SFA intake in humans (Schennink et al., 2007). It is hypothesized that DGAT1 gene polymorphism affects the functional properties of the DGAT1 enzyme (e.g., higher activity or alteration in substrate specificity for different FA), which influences milk FA composition (Grisart et al., 2004; Schennink et al., 2007). Although Grisart et al. (2004) did not show quantitative differences in mRNA expression levels of the DGAT1 gene between alleles, it is not known yet whether DGAT1 gene polymorphism affects the expression of other genes involved in lipid metabolism in the mammary gland. Functional approaches are required to identify effects of DGAT1 gene polymorphism on lipid metabolism and other processes in the cow mammary gland tissue. The objective of this study was to determine the effect of DGAT1 gene polymorphism on the global mRNA expression pattern of genes in the mammary gland tissue of grazing dairy 
cows to get more insight into the effects of this polymorphism on the physiology of the mammary gland.

\section{MATERIALS AND METHODS}

\section{Animals and Diets}

Twenty-eight Holstein-Friesian dairy cows in midlactation (DIM; $176 \pm 22.8 \mathrm{~d}$ ) were used. Cows were fed corn silage, grass silage and concentrates as a mixed ration (MR). In addition to the MR, each cow received $1 \mathrm{~kg}$ of a commercial standard concentrate per day through automatic feeders in the milking parlor. Cows were fed the MR indoors at night, and were grazed on pasture composed of ryegrass (Lolium perenne L.), with approximately $20 \%$ white clover (Trifolium repens L.) during the day. The average paddock size was $5 \mathrm{ha}$, and the stocking rate was 16 cows/ha. Cows were fed the $\mathrm{MR}$ at a level of about $14.5 \mathrm{~kg}$ of $\mathrm{DM} /$ cow per day, and grazed at a daily herbage intake of approximately 5.5 $\mathrm{kg}$ of DM/cow. Ingredients of the concentrates and MR were previously described by Mach et al. (2011). Cows were milked twice per day in the milking parlor at the facility. Two consecutive milk samples (a.m. and p.m. milking) were obtained and pooled. One aliquot was stored at $4^{\circ} \mathrm{C}$ until analysis of fat, protein, and lactose percentage, and another aliquot was frozen at $-20^{\circ} \mathrm{C}$ until analysis for FA composition by gas chromatography, as described by Mach et al. (2011). Approximately 750 to $1,000 \mathrm{mg}$ of mammary tissue from each cow was obtained by surgical biopsy from the midpoint section of a rear quarter according to the method of Farr (Farr et al., 1996). One part of the tissue was used for extraction of DNA and the other for extraction of RNA.

\section{Genotyping}

Prior to genotyping, the quality and quantity of DNA samples isolated from mammary gland tissue were assessed using a NanoDrop spectrophotometer (Isogen Bioscience BV, Maarssen, the Netherlands) and agarose gel electrophoresis. Genotyping of DGAT1 gene polymorphism was performed using a TaqMan allelic discrimination method in an Applied Biosystems 7,500 quantitative real-time (qRT) PCR System (Applied Biosystems, Bleiswijk, the Netherlands), as described by Schennink et al. (2007). The genotype at the DGAT1 locus was designated homozygous Lys (KK), heterozygous Lys/Ala (KA), or homozygous Ala (AA). A kinship coefficient matrix between animals was calculated by using the Kinship package (version 1.1.0.) in the $\mathrm{R}$ programming language (version 2.13; The R Foundation for Statistical Computing, Vienna, Austria), and used to perform 2-way hierarchical cluster analysis (HCA) with "1 - cor (x)" as distance and "average" as aggregation criterion. Additionally, the point-biserial correlation method was applied to the dendrogram to identify the optimum number of clusters as described by Odong et al. (2011). The heatmap function was used to generate images.

\section{RNA Isolation, Processing, and Microarray Analysis}

Total RNA from mammary gland tissue (50 to $100 \mathrm{mg}$ ) was isolated using TRIzol reagent (Invitrogen, Breda, the Netherlands), following the manufacturer's instructions. The RNA purity and concentrations were determined using a NanoDrop ND 1,000 spectrophotometer (Isogen Bioscience BV), and the RNA quality was assessed using a Bioanalyzer 2,100 (Agilent Technologies Netherlands BV, Amsterdam, the Netherlands). The RNA of each biopsy was amplified, biotin labeled, and hybridized to a single-dye Affymetrix GeneChip Bovine Genome Array (\#900493; Affymetrix Inc., Santa Clara, CA) by ServiceXS BV (Leiden, the Netherlands), as described in the user's manual (GeneChip Expression Analysis Technical Manual). For further details, see the Supplemental Materials and Methods (available online at http://www.journalofdairyscience.org/). Arrays were scanned using an Affymetrix GeneChip Scanner $7 \mathrm{G}$ and Affymetrix GeneChip operating software version 1.4, following the GeneChip specifications. After scanning, the Affymetrix GeneChip Command Console (AGCC) software automatically acquired and analyzed image data and computed an intensity value for each probe cell. A total of 28 one-color arrays were prepared, one array per RNA sample. All microarray analyses, including preprocessing quality assessment, normalization, and statistical analysis, were carried out using Bioconductor packages (version 2.7) in the $\mathrm{R}$ programming language (version 2.13). Details on transcriptome data acquisition, quality control, summarization, and normalization are described in Mach et al. (2011). Briefly, data were quality assessed before and after normalization using several built-in quality control methods implemented in the Bioconductor affycoretools and associated packages to identify eventual irregularities of array hybridization, RNA degradation, and data normalization. Arrays were considered of sufficiently high quality if they showed less than $10 \%$ specks in fit probe level model images, if they were not deviating in RNA degradation and density plots, and if they were not significantly deviating in normalized unscaled standard errors (NUSE) and relative log expression (RLE) plots. Upon rigorous examination of the built-in quality control methods to identify ir- 
regularities of array hybridization, RNA degradation, and data normalization, 2 microarrays related to the AA genotype were discarded. Therefore, we included 26 microarrays in our further analysis: 9, 13, and 4 microarrays for AA, KA, and KK genotypes, respectively. Filters were applied to the data to improve the quality of the normalized data set. Probes that did not have a minimum threshold of 40 raw intensity units in at least 1 array per genotype were omitted from analysis. The threshold of 40 was chosen based on an interquartile range (IQR) offset of 0.25 for at least 1 array per genotype (Caesar et al., 2010). This control filtering criteria reduced the number of probes from 24,128 to 7,486 . All microarray experiment data are MIAME compliant and have been deposited in the Gene Expression Omnibus (GEO; http://www.ncbi.nlm.nih.gov/geo/; accession number GSE33720).

\section{Validation of Differential Gene Expression by $q R T-P C R$}

A total of 6 genes differentially expressed between genotypes were validated by qRT-PCR: regulator of G-protein signaling 1 (RGS1), ubiquitin D ( $\boldsymbol{U} \boldsymbol{B D})$, PHD finger protein 3 (PHF3), fibulin 5 (FBLN5), pyruvate dehydrogenase (lipoamide) $\alpha 1$ (PDHA1), and UHRF1-binding protein 1-like (UHRF1BP1L). These genes exhibited the highest amplitude of variation between the genotypes [at least higher than $\pm 40 \%$ between 2 genotypes, absolute value $\log _{2}$ (ratio) $>0.485]$. Reverse transcription of $1 \mu \mathrm{g}$ of the isolated total RNA was performed in a $20-\mu \mathrm{L}$ reaction using Superscript 3 reverse transcriptase (Invitrogen), deoxyribonucleotide triphosphate (dNTP; Roche Diagnostics Nederland BV, Almere, the Netherlands), and random hexamer primers (Roche Diagnostics Nederland BV) for $1 \mathrm{~h}$ at $50^{\circ} \mathrm{C}$ according to the manufacturer protocol (Invitrogen). Templates were amplified after preincubation for $10 \mathrm{~min}$ at $95^{\circ} \mathrm{C}$, followed by amplification for 40 cycles $\left(10 \mathrm{~s}\right.$ at $95^{\circ} \mathrm{C}, 5 \mathrm{~s}$ at $60^{\circ} \mathrm{C}$, and $5 \mathrm{~s}$ at $72^{\circ} \mathrm{C}$ ) on a LightCycler 1.2 qRT-PCR System by using FastStart DNA Master SYBR Green 1 reagents (Roche Diagnostics Nederland BV). The gene-specific primers used are indicated in Supplemental Table S1 (available online at http://www.journalofdairyscience.org/). All reactions revealed a single product as determined by melting curve analysis. Quantitative measurements were performed by establishing a linear amplification curve from 10-fold serial dilutions of cDNA for each gene, and efficiencies of the used sets of primers were at least $95 \%$. Values were calculated according to the comparative cycle threshold $(\mathrm{Ct})$ method using $\beta$-actin $(\boldsymbol{A C T B})$ as the endogenous reference gene.

\section{Statistical Analyses}

Milk quality, FA composition, and qRT-PCR data were analyzed using a mixed-effects model (SAS Institute Inc., Cary, NC). The model included genotype as fixed effect and cow as a random effect. Gene expression was analyzed as described above using the microarray ANOVA (MAANOVA) package (version 1.1.0.) in the $\mathrm{R}$ programming language (version 2.13). The $P$-values were corrected for multiple testing using a false discovery rate $(\mathbf{F D R})$ method $<10 \%(q$-value $<0.10)$, which provides an estimate of the fraction of false discoveries among the significant terms (Bünger et al., 2007). In parallel, the factor analysis for multiple testing (FAMT) method (version 2.20.0) was also applied to analyze gene expression in $\mathrm{R}$ programming (Causeur et al., 2011). The FAMT method is able to remove expression heterogeneity from the data for the subsequent statistical analysis by adjusting the raw data from the common structure. It is specially designed to select differentially expressed genes in microarray data when the correlation structure among gene expression is strong (Causeur et al., 2011). An FDR $<10 \%$ was used as a threshold for significance of differential expression. Two-way HCA was performed using the hclust function with "1 - cor (x)" as distance and "average" as aggregation criterion. The heatmap function was used to generate images. Principal component analysis (PCA) was performed with the FactoMineR library (http:// cran.r-project.org/web/packages/FactoMineR/index.

html). The list of significant genes identified was uploaded into Ingenuity Pathways Analysis (IPA version 5.5; Ingenuity Systems Inc., Redwood City, CA).

Moreover, microarray data was analyzed at the level of differentially expressed functional modules by gene set enrichment analysis (GSEA). Gene set enrichment analysis discovers a collection of genes that show moderate but coordinated differentiation, even when individual genes are not. Therefore, GSEA can identify more subtle changes in expression than the gene list that results from univariate statistical analysis. To that end, the GSEA method first calculated for each group of genes an enrichment score (ES) that reflected the degree of association between the gene expression and the genotypes. Then, it estimated the significance level of the ES by using an empirical phenotype-based permutation test procedure that preserved the complex correlation structure of the gene expression data, and lastly an adjustment for multiple hypothesis testing was done (Subramanian et al., 2005). Gene set size filter considered a minimum of 15 and a maximum of 500 genes, and the number of permutations was set to 1,000. Gene sets were considered significantly enriched at an 
Table 1. Effect of diacylglycerol-O-transferase 1 (DGAT1) gene polymorphism on milk yield and milk composition

\begin{tabular}{lccccc}
\hline & \multicolumn{5}{c}{ Genotype $^{1}$} \\
\cline { 2 - 3 } Trait & AA $(\mathrm{n}=9)$ & KA $(\mathrm{n}=13)$ & KK $(\mathrm{n}=4)$ & SEM & $P$-value \\
\hline DIM & 189 & 171 & 167 & 22.8 & 0.85 \\
Milk production $(\mathrm{kg} / \mathrm{d})$ & 24.8 & 20.8 & 22.6 & 1.68 & 0.25 \\
Fat content (\%) & 4.16 & 4.34 & 4.61 & 0.160 & 0.50 \\
Fat yield (kg/d) & 0.99 & 0.90 & 1.11 & 0.081 & 0.48 \\
Protein content (\%) & 3.70 & 3.81 & 3.63 & 0.122 & 0.72 \\
Protein yield (kg/d) & 0.88 & 0.79 & 0.88 & 0.074 & 0.69 \\
Lactose content (\%) & 4.28 & 4.45 & 4.36 & 0.090 & 0.51 \\
Lactose yield $(\mathrm{kg} / \mathrm{d})$ & 1.01 & 0.92 & 1.07 & 0.071 & 0.61 \\
\hline
\end{tabular}

${ }^{1}$ The genotype at the DGAT1 232 locus was designated KK, KA, or AA for homozygous Lys, heterozygous Lys/Ala, or homozygous Ala, respectively.

FDR <0.05. Normalized ES of significantly enriched pathways between genotypes groups were calculated.

\section{RESULTS}

\section{Genotypes and Allele Frequencies}

The frequency of the AA, KA, and KK genotype was $0.46,0.40$, and 0.14 , respectively. The observed genotype frequencies are consistent with those from Holstein-Friesian populations in the Netherlands (Schennink et al., 2007), the UK (Banos et al., 2008), Ireland (Berry et al., 2010), and France (Gautier et al., 2007). The minor allele frequency $(\mathrm{K})$ was 0.37 , similar to that reported by Schennink et al. (2007) in the Netherlands. Additionally, the kinship coefficients were $6.9,5.5$, and $1.7 \%$ for $\mathrm{AA}, \mathrm{KA}$, and $\mathrm{KK}$ genotypes, respectively. Values observed for AA and KA genotypes are consistent with those expected in the Dutch Holstein-Friesian population (5.27\%; Danchin-Burge et al., 2011). The discrepancy in the level of kinship coefficient in the KK animals compared with AA and KA genotypes is probably attributed to sample size and differences in the depth of the pedigree available, as 2 of the animals came from Germany and not all the information was available. The kinship coefficient matrix is plotted in Supplemental Figure S1, whereas the resulting heatmap and dendrogram are presented in Supplemental Figures S2 and S3 (available online at http://www.journalofdairyscience.org/), respectively. The point-biserial correlation was not able to identify a significant number of clusters $\left(\mathrm{R}^{2}=0.10 ; P=0.98\right)$. The latest result, together with the off-diagonal entries in Supplemental Figure S1 (available online at http:// www.journalofdairyscience.org/), suggests that no global population structure exists within and between genotypes.

\section{Milk Production and Composition}

Substituting A for $\mathrm{K}$ at the A232K DGAT1 locus numerically decreased milk yield and increased fat yield, although these effects were not significant (Table 1). With regard to the FA composition (Table 2), the proportion of $\mathrm{C} 14: 0$ was greater $(P=0.04)$ in AA genotypes as compared with $\mathrm{KA}$ and $\mathrm{KK}$ genotypes.

Table 2. Effect of diacylglycerol- $O$-transferase 1 (DGAT1) gene polymorphism on milk FA composition (g/100 $\mathrm{g}$ of total FA)

\begin{tabular}{lccccc}
\hline & \multicolumn{4}{c}{ Genotype $^{1}$} \\
\cline { 2 - 4 } FA & AA $(\mathrm{n}=9)$ & $\mathrm{KA}(\mathrm{n}=13)$ & $\mathrm{KK}(\mathrm{n}=4)$ & SEM & $P$-value \\
\hline C4:0 & 2.74 & 2.73 & 2.75 & 0.110 & 0.98 \\
C6:0 & 2.14 & 2.11 & 2.15 & 0.070 & 0.95 \\
C8:0 & 1.44 & 1.37 & 1.47 & 0.051 & 0.66 \\
C10:0 & 3.25 & 3.02 & 3.3 & 0.142 & 0.51 \\
C11:0 & 0.43 & 0.44 & 0.50 & 0.022 & 0.41 \\
C12:0 & 4.04 & 3.73 & 4.08 & 0.142 & 0.32 \\
iso C13:0 & 0.02 & 0.02 & 0.02 & 0.004 & 0.79 \\
anteiso C13:0 & 0.11 & 0.11 & 0.14 & 0.009 & 0.26 \\
C13:0 & 0.20 & 0.21 & 0.26 & 0.012 & 0.16 \\
C14:0 & 11.82 & 11.13 & 11.26 & 0.173 & $*$
\end{tabular}


Table 2 (Continued). Effect of diacylglycerol-O-transferase 1 (DGAT1) gene polymorphism on milk FA composition $(\mathrm{g} / 100 \mathrm{~g}$ of total FA)

\begin{tabular}{|c|c|c|c|c|c|}
\hline \multirow[b]{2}{*}{ FA } & \multicolumn{3}{|c|}{ Genotype $^{1}$} & \multirow[b]{2}{*}{ SEM } & \multirow[b]{2}{*}{$P$-value } \\
\hline & $\mathrm{AA}(\mathrm{n}=9)$ & $\mathrm{KA}(\mathrm{n}=13)$ & $\mathrm{KK}(\mathrm{n}=4)$ & & \\
\hline iso $\mathrm{C} 15: 0$ & 0.22 & 0.21 & 0.22 & 0.004 & $\dagger$ \\
\hline anteiso $\mathrm{C} 15: 0$ & 0.50 & 0.49 & 0.48 & 0.013 & 0.87 \\
\hline cis-9 C14:1 & 1.22 & 1.34 & 1.50 & 0.090 & 0.42 \\
\hline C15:0 & 1.02 & 1.07 & 1.22 & 0.030 & $\dagger$ \\
\hline iso $\mathrm{C} 16: 0$ & 0.20 & 0.17 & 0.17 & 0.008 & 0.11 \\
\hline C16:0 & 28.47 & 29.47 & 30.85 & 0.652 & 0.25 \\
\hline iso $\mathrm{C} 17: 0$ & 0.16 & 0.16 & 0.15 & 0.007 & 0.88 \\
\hline cis-9 C16:1 & 1.62 & 1.96 & 2.24 & 0.090 & $* *$ \\
\hline anteiso $\mathrm{C} 17: 0$ & 0.27 & 0.21 & 0.23 & 0.012 & 0.13 \\
\hline C17:0 & 0.61 & 0.61 & 0.63 & 0.012 & 0.75 \\
\hline cis-9 C17:1 & 0.16 & 0.19 & 0.20 & 0.012 & 0.14 \\
\hline C18:0 & 9.94 & 8.82 & 8.16 & 0.451 & 0.16 \\
\hline trans-4 C18:1 & 0.01 & 0.01 & 0.01 & 0.002 & 0.85 \\
\hline trans $-5 \mathrm{C} 18: 1$ & 0.01 & 0.01 & 0.01 & 0.002 & 0.54 \\
\hline trans- $6+$ trans-8 C18:1 & 0.29 & 0.32 & 0.24 & 0.010 & 0.20 \\
\hline trans-9 C18:1 & 0.16 & 0.19 & 0.17 & 0.011 & 0.49 \\
\hline trans-10 + trans-11 C18:1 & 1.38 & 1.80 & 1.72 & 0.122 & 0.14 \\
\hline trans-12 C18:1 & 0.41 & 0.46 & 0.39 & 0.013 & 0.11 \\
\hline trans $-13+$ trans -14 C18:1 & 0.32 & 0.34 & 0.40 & 0.027 & 0.42 \\
\hline cis-12 C18:1 & 0.06 & 0.08 & 0.07 & 0.013 & 0.34 \\
\hline cis-9 C18:1 & 19.55 & 19.27 & 17.64 & 0.541 & 0.26 \\
\hline cis-13 C18:1 & 0.06 & 0.08 & 0.07 & 0.005 & 0.34 \\
\hline trans- $16+$ cis-14 C18:1 & 0.55 & 0.55 & 0.50 & 0.022 & 0.34 \\
\hline cis-15 C18:1 & 0.24 & 0.27 & 0.25 & 0.013 & 0.43 \\
\hline cis-11 C18:1 & 0.30 & 0.35 & 0.35 & 0.033 & 0.15 \\
\hline trans -9, trans-12 C18:2 & 0.007 & 0.008 & 0.016 & 0.002 & 0.35 \\
\hline trans-10,cis-12 C18:2 & 0.01 & 0.01 & 0.01 & 0.001 & 0.58 \\
\hline cis-9, cis-12 C18:2 & 1.48 & 1.57 & 1.38 & 0.040 & $*$ \\
\hline cis-9,trans-11 C18:2 & 0.57 & 0.67 & 0.64 & 0.050 & 0.40 \\
\hline cis-9,cis-12,cis-15 C18:3 & 0.54 & 0.56 & 0.53 & 0.032 & 0.68 \\
\hline cis-6, cis-9, cis-12 C18:3 & 0.02 & 0.02 & 0.01 & 0.002 & 0.74 \\
\hline $\mathrm{C} 20: 0$ & 0.12 & 0.11 & 0.11 & 0.006 & 0.73 \\
\hline cis-11 C20:1 & 0.03 & 0.04 & 0.02 & 0.003 & $*$ \\
\hline cis-11,cis-14 C20:2 & 0.02 & 0.02 & 0.01 & 0.002 & 0.35 \\
\hline cis-11,cis-14,cis-17 C20:3 & 0.01 & 0.01 & 0.01 & 0.002 & 0.53 \\
\hline cis-8,cis-11,cis-14 C20:3 & 0.06 & 0.06 & 0.06 & 0.005 & 0.99 \\
\hline all cis-5, -8, -11, -14 C20:4 & 0.09 & 0.10 & 0.11 & 0.041 & 0.23 \\
\hline all cis- $5,-8,-11,-14,-17 \mathrm{C} 20: 5$ & 0.05 & 0.05 & 0.04 & 0.003 & 0.86 \\
\hline $\mathrm{C} 22: 0$ & 0.04 & 0.05 & 0.04 & 0.003 & 0.36 \\
\hline cis-13 C22:1 & 0.01 & 0.01 & 0.01 & 0.003 & 0.31 \\
\hline cis-13,cis-16 C22:2 & 0.04 & 0.05 & 0.04 & 0.002 & 0.60 \\
\hline all cis- $7,-10,-13,-16$ C22:4 & 0.01 & 0.02 & 0.01 & 0.002 & 0.49 \\
\hline C23:0 & 0.01 & 0.02 & 0.01 & 0.002 & 0.61 \\
\hline all cis-7, $-10,-13,-16,-19$ C22:5 & 0.07 & 0.07 & 0.09 & 0.003 & 0.12 \\
\hline all cis- $4,-7,-10,-13,-16,-19$ C22:6 & 0.01 & 0.01 & 0.01 & 0.002 & 0.72 \\
\hline C24:0 & 0.04 & 0.03 & 0.03 & 0.009 & 0.56 \\
\hline cis-15 C24:1 & 0.01 & 0.01 & 0.01 & 0.001 & 0.74 \\
\hline Unidentified & 2.83 & 3.20 & 3.04 & 0.012 & 0.86 \\
\hline De novo $\mathrm{FA}^{2}$ & 27.4 & 26.2 & 27.4 & 0.56 & 0.35 \\
\hline C16 & 30.0 & 31.4 & 33.9 & 0.64 & 0.26 \\
\hline SFA & 67.7 & 66.2 & 68.2 & 0.97 & 0.49 \\
\hline $\mathrm{LCFA}^{3}$ & 36.5 & 36.1 & 33.2 & 0.76 & 0.11 \\
\hline MUFA & 26.4 & 27.3 & 25.8 & 0.83 & 0.56 \\
\hline $\mathrm{UFA}^{4}$ & 29.4 & 30.5 & 28.8 & 0.89 & 0.50 \\
\hline PUFA & 2.9 & 3.2 & 2.9 & 0.11 & 0.18 \\
\hline Total trans-FA & 3.1 & 3.7 & 3.5 & 0.20 & 0.19 \\
\hline
\end{tabular}

${ }^{1}$ The genotype at the DGAT1 232 locus was designated KK, KA, or AA for homozygous Lys, heterozygous Lys/Ala, or homozygous Ala, respectively.

${ }^{2}$ De novo FA include all FA from $\mathrm{C} 4$ to $\mathrm{C} 14$.

${ }^{3} \mathrm{LCFA}=$ long-chain $\mathrm{FA}$ (includes all $\mathrm{FA} \geq \mathrm{C} 18$ ).

${ }^{4}$ Unsaturated FA.

$\dagger P<0.10 ;{ }^{*} P<0.05 ;{ }^{*} P<0.01$. 
In contrast, KK genotypes lead to an increase $(P=$ 0.01 ) in the proportion of cis-9 C16:1 FA, whereas they lead to a decrease $(P=0.03)$ in the proportion of cis-9,cis-12 C18:2 and cis-1 C20:1 compared with AA and KA genotypes. No significant differences could be observed for the other FA.

\section{Differential Expression of Individual Genes in the Mammary Gland}

Genes identified by MAANOVA did not meet the threshold for statistical significance after multiple hypotheses testing correction. However, the FAMT method, which adjusts data from heterogeneity components, revealed 30 annotated genes, which differed between genotypes (FDR $q$-values $<0.10)$. The gene identification, symbol and description of the 30 resulting genes are shown in Table 3. A 2-way HCA was applied to the normalized data set to identify clusters between the different genotypes and the 30 differently expressed genes (Figure 1). The heatmap separated the heterozygous KA animals from the homozygous AA and KK animals. For the gene variables, 2 clusters were identified: cluster 1 (16 genes) and 2 (14 genes). The majority of genes in cluster 1 were significantly downregulated when comparing $\mathrm{KK}$ and AA genotypes to KA genotypes. On the contrary, the majority of genes in cluster 2 were downregulated when comparing KA genotypes to KK and AA genotypes. Complementary to these results, the PCA allowed us to identify the genes contributing most to the separation of the different clusters (Figure 2). As highlighted in Figure $2 \mathrm{~A}: 1$, the first component, which explains the largest contribution to variability in the gene expression data; that is, retains the most information, clearly separates between gene expression patterns of the heterozygous KA animals from the homozygous AA and KK animals. This first component of PCA explained $41.64 \%$ of the total variability in gene expression. The second component explained $15.53 \%$ and the third $7.91 \%$ (Figure 2A:2), which leads to an improved clustering of the KK genotypes. Variable graphs (Figure 2B:1 and 2B:2) have been generated to better understand genes that contribute the most to separate expression patterns of the heterozygous KA animals from the homozygous AA and KK animals. In Figure 2B:1, the quality of representation of a variable on the axis is measured by the squared cosine between the vector issued from the element and its projection on the axis. If this square cosine is close to 1 , it means that the element is well projected on the axis (Lê et al., 2008). Projecting the arrows onto the first dimension (Figure 2B:1) we can see that PDHA1, RMB18, TMEM9, and LOC521568, and PHF3, ATL2, and ZBTB38 are the most important for the first component. For the third principal component (Figure 2B:2), the gene FBLN5 and C13H20ORF43 are the most important. Over $85 \%$ of the genes showed a high correlation with the first 3 principal components ( $\mathrm{r}$ $>0.6$ ). In this context, most of the genes contributed to split-up genotypes into the 2 main clusters represented in Figure 2A (homozygous vs. heterozygous). Furthermore, the RGS1, UBD, PHF3, FBLN5, PDHA1, and $U H R F 1 B P 1 L$ genes not only presented correlation coefficient $>0.6$ with respect to the principal components represented, but also exhibited the highest amplitude of variation between the genotypes [at least higher than $\pm 40 \%$, absolute value $\log _{2}$ (ratio) $\left.>0.485\right]$. These genes are indicated in black in Figure 2B. Therefore, they were used to validate the microarray gene expression by qRT-PCR (Supplemental Figure S4, available online at http://www.journalofdairyscience.org/). Overall, the qRT-PCR data confirmed the microarray data. The Ingenuity Pathways Analysis of the 30 genes revealed that they encode for molecular functions involved in growth, proliferation and development, signaling, molecular transport, cell death modulation, assembly and organization, as well as immune system and inflammatory response (Supplemental Table S2, available online at http://www.journalofdairyscience.org/).

\section{Differential Expression of Functional Modules in the Mammary Gland}

Instead of analyzing the differential expression of individual genes, we also analyzed the microarray data at the level of gene sets that together encode for particular functional modules (Figure 3). Notably, the gene sets involved in energy metabolism (oxidative phosphorylation, electron transport chain, citrate cycle, propionate metabolism, and lipid biosynthesis) were enriched in KK and KA compared with AA genotypes. Additionally, genes sets related to immune response were affected when comparing KK with $\mathrm{KA}$ and $\mathrm{AA}$ genotypes (i.e., IL-7 signaling, and antigen processing and presentation). The cell communication processes were overrepresented when comparing the AA and KK genotypes.

\section{DISCUSSION}

\section{Milk Production and Composition}

The K allele was associated with numerically reduced milk production and increased milk fat yield, consistent with previous work in dairy cattle (Schennink et al., 2007; Banos et al., 2008; Berry et al., 2010). Absence of statistically significant effects in the present analysis almost certainly relates to the limited sample size. 
Table 3. Expression of differentially expressed genes identified by using factor analysis for multiple testing method when comparing the effect of diacylglycerol- $O$-transferase 1 (DGAT1) gene polymorphism on the global expression pattern of genes in the mammary gland tissue of dairy cows ${ }^{1}$

\begin{tabular}{|c|c|c|c|c|c|c|c|}
\hline \multirow[b]{2}{*}{ Identity of gene } & \multirow[b]{2}{*}{ Gene symbol } & \multirow[b]{2}{*}{ Description } & \multicolumn{3}{|c|}{ Genotype $^{2}$} & \multirow[b]{2}{*}{ SEM } & \multirow[b]{2}{*}{$q$-value } \\
\hline & & & $\mathrm{AA}$ & $\mathrm{AK}$ & KK & & \\
\hline Bt.12614.1.A1_s_at & EIF3B & Eukaryotic translation initiation factor 3 , subunit B & 63.9 & 103.8 & 68.6 & 7.13 & $\dagger$ \\
\hline Bt.13063.1.S1_at & $U B E 2 A$ & Ubiquitin conjugating enzyme E2A & 130.3 & 157.4 & 129.2 & 4.34 & $\dagger$ \\
\hline Bt.1370.1.S1_at & $A S S 1$ & Argininosuccinate synthetase 1 & 138.3 & 189.2 & 146.1 & 9.81 & $\dagger$ \\
\hline Bt.14993.2.A1_at & ATL2 & Atlastin guanosine triphosphatase (GTPase) 2 & 89.6 & 75.2 & 97.3 & 3.43 & $\dagger$ \\
\hline Bt.17703.1.S1_at & SLC15A2 & Solute carrier family $15\left(\mathrm{H}^{+} /\right.$peptide transporter $)$, member 2 & 30.8 & 54.9 & 31.3 & 4.47 & $\dagger$ \\
\hline Bt.19415.1.A1_at & PDHA1 & Pyruvate dehydrogenase (lipoamide) $\alpha 1$ & 35.0 & 69.8 & 42.6 & 4.77 & * \\
\hline Bt.19938.1.A1_at & SP3 & Predicted: hypothetical LOC540766 (LOC540766) & 514.0 & 431.0 & 556.1 & 16.20 & * \\
\hline Bt.20213.2.S1_at & RBM18 & RNA-binding motif protein 18 (RBM18) & 188.8 & 248.9 & 169.1 & 9.65 & * \\
\hline Bt.20400.2.S1_at & LOC521568 & Similar to ATP-binding cassette, subfamily C, member 4 & 338.7 & 440 & 352 & 14.9 & $\dagger$ \\
\hline Bt.20639.1.A1_at & PHF3 & Predicted: similar to KIAA0244 (LOC522928) & 43.4 & 22.2 & 52.8 & 3.75 & * \\
\hline Bt.20938.1.S1_at & ZВТВ38 & Zinc finger and BTB domain-containing 38 & 150.9 & 116.3 & 149.1 & 4.8 & $\dagger$ \\
\hline Bt.21503.1.S1_at & PPP1CA & Protein phosphatase 1 , catalytic subunit, $\alpha$ isoform & 222.3 & 258.1 & 225.0 & 7.82 & $\dagger$ \\
\hline Bt.22594.1.S1_at & LOC789894 & $\begin{array}{l}\text { Similar to PAP-associated domain-containing protein } 5 \\
\text { (topoisomerase-related function protein } 4-2 ; \text { TRF4-2) }\end{array}$ & 323.3 & 264.1 & 370.7 & 10.80 & * \\
\hline Bt.23273.1.S1_at & $C I R B P$ & Cold-inducible RNA-binding protein & 248.2 & 323.3 & 276.3 & 20.12 & $\dagger$ \\
\hline Bt.2374.1.S1_at & GNB1 & Guanine nucleotide-binding protein ( $\mathrm{G}$ protein), $\beta$ polypeptide 1 & 403.3 & 471.4 & 422.3 & 16.13 & $\dagger$ \\
\hline Bt.24580.1.A1_at & TMEM9 & Transmembrane protein 9 & 41.5 & 56.3 & 34.4 & 2.58 & $\dagger$ \\
\hline Bt.27024.1.A1_at & CCAR1 & Cell division cycle and apoptosis regulator 1 & 716.3 & 565.1 & 858.1 & 34 & * \\
\hline Bt.28269.1.S1_at & RHOJ & Ras homolog gene family, member J & 120.0 & 119.0 & 87.3 & 6.6 & $\dagger$ \\
\hline Bt.29411.1.S1_at & UHRF1BP1L & Hypothetical LOC520720 (LOC520720) & 58.4 & 32.8 & 70.19 & 4.68 & * \\
\hline Bt.3399.1.S1_a_at & $L H P P$ & $\begin{array}{l}\text { Phospholysine phosphohistidine inorganic } \\
\text { pyrophosphate phosphatase }\end{array}$ & 66.1 & 55.9 & 70.5 & 2.79 & $\dagger$ \\
\hline Bt.3539.1.S1_at & C13H20ORF43 & Chromosome 20 open reading frame 43 ortholog & 74.1 & 72.1 & 58.3 & 1.88 & $\dagger$ \\
\hline Bt.425.1.S2_at & $S L T M$ & $\begin{array}{l}\text { Scaffold attachment factor B (SAFB)- } \\
\text { like, transcription modulator }\end{array}$ & 188.9 & 172.2 & 225.5 & 5.56 & * \\
\hline Bt.4310.1.A1_at & $R G S 1$ & Regulator of G-protein signaling 1 & 54.6 & 35.9 & 161.1 & 9.41 & * \\
\hline Bt.5709.1.S1_at & FGFR1OP2 & Fibroblast growth factor receptor 1 (FGFR1) oncogene partner 2 & 237.4 & 200.2 & 203.2 & 9.36 & $\dagger$ \\
\hline Bt.5897.1.S1_at & $U B D$ & Similar to ubiquitin D, transcript variant 2 (LOC512938) & 224.0 & 165.5 & 385.0 & 32.40 & * \\
\hline Bt.6449.1.S1_at & FBLN5 & Fibulin 5 & 88.0 & 66.0 & 45.9 & 6.97 & $*$ \\
\hline Bt.7413.1.S1_at & $G R N$ & Granulin & 59.4 & 76.4 & 60.6 & 2.86 & $\dagger$ \\
\hline Bt.7505.1.S1_at & STK38 & Serine/threonine kinase 38 & 261.0 & 217.0 & 310.1 & 10.54 & $\dagger$ \\
\hline Bt.8243.1.A1_at & ZFP91 & $\begin{array}{l}\text { Similar to zinc finger protein homologous } \\
\text { to mouse Zfp91 (LOC529006) }\end{array}$ & 90.9 & 66.9 & 94.9 & 4.57 & $\dagger$ \\
\hline Bt.9031.1.S1_at & PTBP1 & Polypyrimidine tract-binding protein 1 & 42.0 & 71.0 & 44.9 & 4.19 & $\dagger$ \\
\hline
\end{tabular}

${ }^{1}$ A cut-off of false discovery rate $q$-values $<0.10$ was used.

Ф ${ }^{2}$ The genotype at the DGAT1 232 locus was designated KK, KA, and AA for homozygous Lys, heterozygous Lys/Ala, and homozygous Ala, respectively.

으 $\quad \dagger q$-value $<0.10 ;{ }^{*} q$-value $<0.05$. 


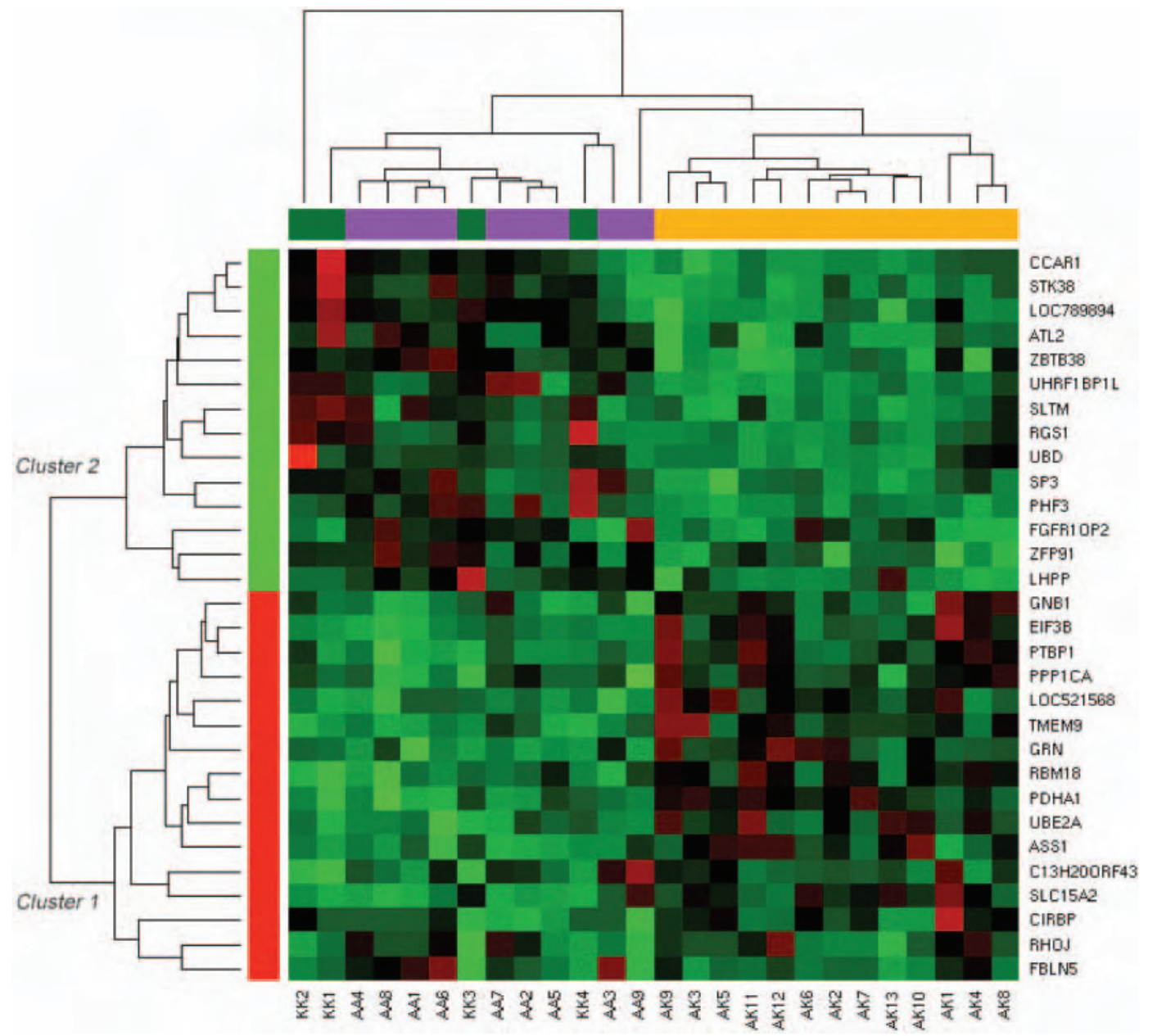

Figure 1. Two-way hierarchical clustering analysis of the genes identified by factor analysis for multiple testing method $(q$-values $<0.10)$ when comparing the effect of the diacylglycerol- $O$-transferase 1 gene polymorphism on the global expression pattern of genes in the mammary gland tissue of dairy cows. The color-coded gene module is displayed in the color bars to the left of the dendrograms. Each column in the heatmap corresponds to 1 animal (labeled by color: green $=$ homozygous Lys, violet $=$ homozygous Ala, and orange = heterozygous Lys/Ala). In the heatmap, the green color represents low adjacency (negative correlation), whereas red represents high adjacency (positive correlation). Gene clusters 1 and 2 are displayed to the left of the dendrograms.

Nevertheless, the KK genotype was associated with a higher proportion of cis-9 C16:1 FA in milk fat, and a lower proportion of cis9,cis 12 C18:2 and cis-1 20:1 compared with the AA and KA genotypes. Furthermore, in agreement to Schennink et al. (2007), the KK genotype was associated with lower proportions of C14:0. The observed effects of DGAT1 gene polymorphism on milk FA composition might be explained by a higher activity of DGAT1 enzyme and alteration of substrate specificity of DGAT1, as suggested previously (Grisart et al., 2004; Schennink et al., 2007). These observations, together with several gene expression effects (see next sections) are the major factor in leading us to believe that DGAT1 polymorphism was indeed the main factor affecting milk yield and composition, rather than another mutation located in the same chromosome 14 that would be in strong linkage disequilibrium with K232A.

\section{Differential Expression of Genes in the Mammary Gland}

The present study is, to our knowledge, the first one in which microarrays were used for analysis of the bovine mammary gland transcriptome in relation to polymorphism in the exon region of the DGAT1 gene. The analysis with the MAANOVA did not identify individual genes that met the threshold for statistical significance. The differences in gene expression were probably modest relative to the experimental and biological noise, which is inherent to technical features of the experiment (RNA isolation and handling, chip 
A: 1)

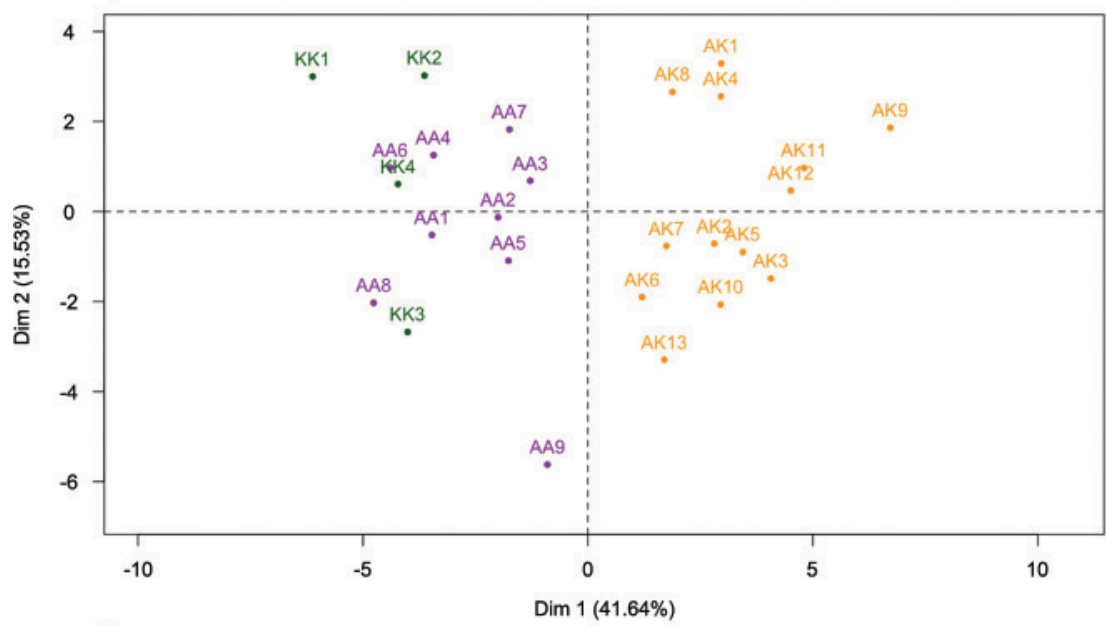

2)

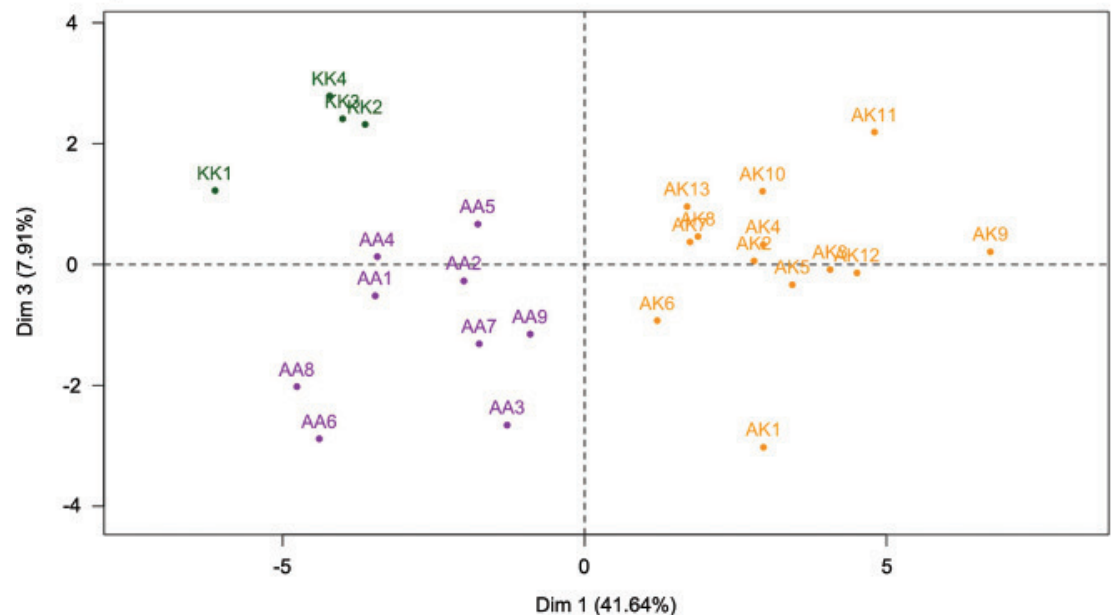

B: 1)

PCA 1×2, scaled variables

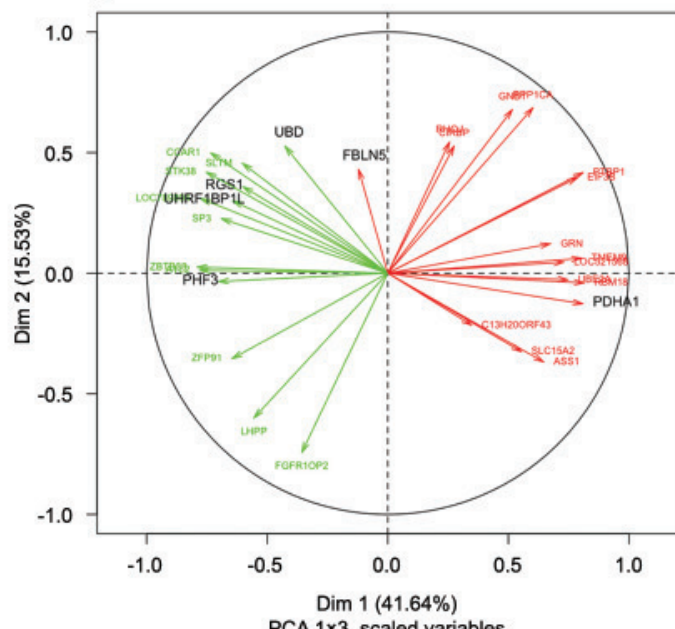

2)

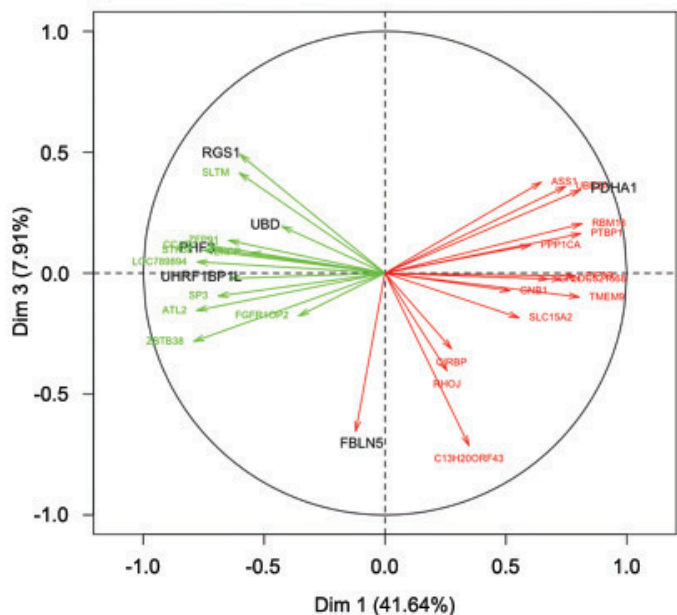

Figure 2. Principal component analysis $(\mathrm{PCA})$ of genes identified by factor analysis for multiple testing method $(q$-values $<0.10)$ when comparing the effect of the diacylglycerol-O-transferase 1 (DGAT1) gene polymorphism on the global expression pattern of genes in the mammary gland tissue of dairy cows. A: animal factor map; A:1 is a projection in the X1-X2 plane; :2 is a projection in the X1-X3 plane. Animals are labeled by color: green $=$ homozygous Lys, violet $=$ homozygous Ala, and orange $=$ heterozygous Lys/Ala. The contribution of each animal to the construction of 1 dimension (Dim) allows detection among the genotypes of which ones are extreme and contribute to the construction of the dimension. B: genes factor map; B:1 is a projection in the X1-X2 plane; B:2 is a projection in the X1-X3 plane. The 30 genes identified by factor analysis for the multiple testing method that contribute to the construction of the axes are projected. The quality of representation of a gene on the axis of rank $s$ is measured by the squared cosine between the vector issued from the element and its projection on the axis. If this square cosine is close to 1, it means that the element is well projected on the axis (Lê et al., 2008). The quality of representation of a gene on a plane can be visualized by the distance between the projected variable onto the plane and the correlation circle (circle of radius 1 ). The genes that appeared to be highly affected by the DGAT1 gene polymorphism (at least higher than $\pm 40 \%$ between 2 genotypes, absolute value $\log _{2}($ ratio) $>0.485$; results in Table 3 ) are indicated in black.

hybridization conditions, and scanner characteristics) and to gene-environment interactions, independent of DGAT1 gene polymorphism effect. In contrast to MAANOVA, the more advanced FAMT approach allowed us to identify 30 annotated genes whose expression in the mammary gland differed significantly between the DGAT1 genotypes. The FAMT method is able to capture and remove the hidden dependence structure independent of DGAT1 gene polymorphism and offers improved high-dimensional multiple testing procedures (Friguet et al., 2009; Blum et al., 2010). The FAMT method removes expression heterogeneity from the data before subsequent analysis of statistical significance by modeling the common information shared by all the genes using a factor analysis structure (Blum et al., 2010; Causeur et al., 2011). As the uncontrolled effects and technological biases are extracted from the statistical noise, FAMT shows an improved capacity to test differential expression of gene expression compared with MAANOVA. 


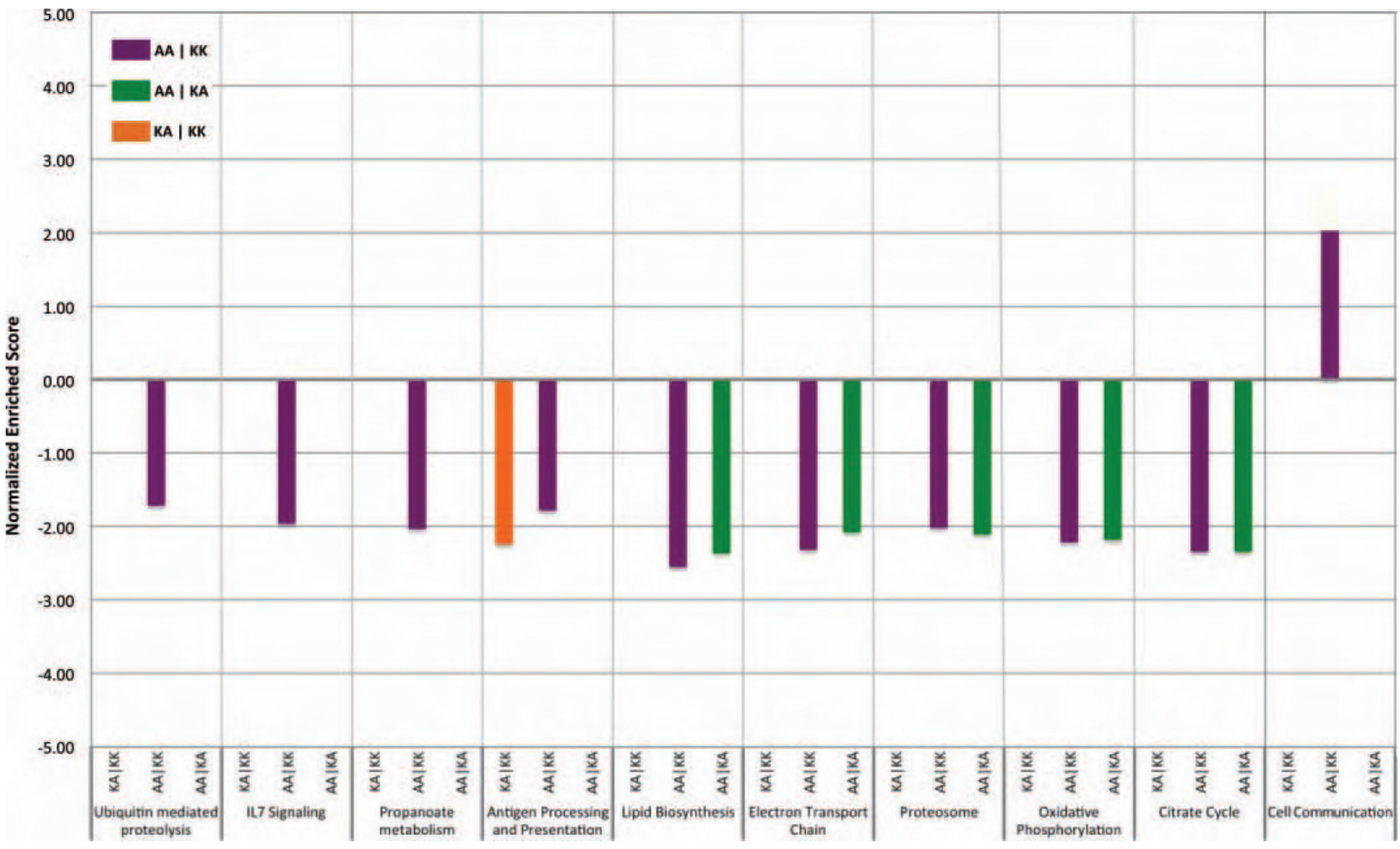

Figure 3. Normalized enriched scores (NES) of enriched gene sets $(q$-values $<0.05)$ in the mammary gland of dairy cows identified by using the gene set enrichment analysis method when comparing the different diacylglycerol- $O$-transferase 1 (DGAT1) genotypes. Gene sets were considered significantly enriched at a false discovery rate $q$-value $<5 \%$. Comparisons between genotypes are labeled by color: green $=\mathrm{AA} \mid \mathrm{KA}$, violet $=\mathrm{AA} \mid \mathrm{KK}$, and orange $=\mathrm{KA} \mid \mathrm{KK}$. The genotype at the DGAT1 232 locus was designated KK, KA, or AA for homozygous Lys, heterozygous Lys/Ala, or homozygous Ala, respectively. The value of the NES reflects the degree to which a gene set is overrepresented at the top or bottom of a ranked list of genes. A positive NES indicates gene set enrichment at the top of the ranked list; a negative NES indicates gene set enrichment at the bottom of the ranked list. Color version available in the online PDF.

A finding of particular interest was that by using the HCA and PCA approaches, the analyzed groups of animals could be split into homozygous and heterozygous genotypes based on their overall gene-expression profiles. Compared with homozygous animals, heterozygous DGAT1 animals presented a downregulation of genes that mainly play a role in cellular signaling and immune responses. On the other hand, the heterozygous genotype presented substantial upregulation of genes related to the organization of the mammary gland tissue. Whether this also leads to differences in the cellular and functional dynamics of mammary tissue between homozygous and heterozygous animals is not yet known.

Notably, out of the identified 30 annotated genes whose expression in the mammary gland differed significantly between the DGAT1 genotypes, RGS1 and $U B D$ genes exhibited the 2 highest expressions in the genotype KK compared with the $\mathrm{AA}$ and KA genotypes. Evidence exists that $R G S 1$ regulates cell signal- ing, which is accomplished by a myriad of proteins, lipids, ions, and small molecules (McCudden et al., 2005). Donaldson et al. (2005) described that RGS1 may play a role in the mammary gland of dairy cows in attenuating of the Ras-mediated signal that promotes the activity of peripheral blood lymphocytes. Moreover, Connor et al. (2008) reported that $U B D$ is involved in immune responses and inflammation in the mammary gland of dairy cows. The exact molecular function of these genes in mammary tissue of dairy cows remains to be elucidated.

\section{Differential Expression of Functional Modules in the Mammary Gland}

In line with the individual gene-based analysis, GSEA showed that DGAT1 gene polymorphism modified the expression of sets of genes that mainly modulate cell signaling, cell energy metabolism, and immune system response. Remarkably, the gene sets involved in energy 
metabolism (oxidative phosphorylation, electron transport chain, citrate cycle, and lipid biosynthesis) were less expressed in AA compared with KK and KA genotypes. The gene sets featured several functional related genes that play a crucial role in TAG synthesis, such as glycerol-3-phosphate acyltransferase mitochondrial $(G P A M)$, 1-acylglycerol-3-phosphate $O$-acyltransferase 4 (AGPAT4), and insulin-induced gene 1 (INSIG1). Also, several genes involved in lipid metabolism [i.e., FA synthase $(F A S N)$ and stearoyl-CoA desaturase 1 (SCD1)] were found to be downregulated in AA compared with KA and KK genotype animals. Although none of those individual genes in the pathway met the threshold for statistical significance after adjustment for multiple testing between genotypes (FDR $<0.10$ ), the pathway did. This suggested that pathway approaches can identify genes that show moderated but consistent and coordinated differentiation in expression levels. Furthermore, this pattern of gene expression suggests that compared with the $\mathrm{K}$ allele, the A allele is associated with reduced energy metabolism. Although the set of lipid metabolism genes in AA genotyped cows was downregulated, their milk fat content was only numerically lower than in KK genotyped cows, probably as a result of the limited sample size used.

It is noteworthy that we also identified immune system pathways that were sensitive to DGAT1 polymorphism. Such an effect of DGAT1 gene polymorphism in the mammary gland in dairy cows has never been reported before. Cows with the KK genotype revealed enrichment in gene sets known to be involved in immune responses compared with the AA and KA genotypes. It featured several genes involved in histocompatibility complex class II. Some of these immune related genes, including genes encoding complement component $5 \mathrm{a}$ re-

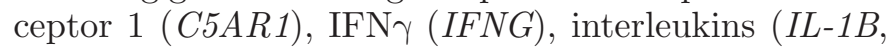
$I L-8, I L-6$, and $I L-8 R A)$, LPS-binding protein $(L B P)$ and CD14 antigen (CD14), have been found associated with bovine mastitis (Ogorevc et al., 2009). Our observations indicate that DGAT1 gene polymorphism relates to modifications in the immune response transcripts. The mechanism for these changes in immune response gene expressions in the mammary gland is unknown and requires further investigation. The current findings raise an interesting correspondence with those obtained for lipid mobilization in transition dairy cows. Contreras and Sordillo (2011) suggested that in transition dairy cows, excessive accumulation of lipid components in the hepatocytes and other cells could cause physical damage, including compression and reduction in size and number of organelles, and could induce programmed cell death or apoptosis, intracellular signaling, and alter the expression of proinflammatory mediators through lipid mediator biosynthetic path- ways. Based on the observed effects, it is likely that DGAT1 polymorphism affects the concentration and composition of TAG accumulated in the matrix and lumen of the mammary gland (Koliwad et al., 2010). In turn, this accumulation may not only alter cellular biosynthesis pathways related to lipid metabolism, but also modifies tissue-based processes involved in immune system response or tissue remodeling. This intriguing possibility remains to be demonstrated, although Loor et al. (2005) and Loor et al. (2006) suggested a causal link between hepatic inflammation and liver TAG accumulation in periparturient dairy cows through the activation of genes that have important roles in aspects of cell proliferation and immune response. Additionally, Bionaz et al. (2012) recently reported that also in bovine kidney cells, saturated long-chain FA not only modulate the expression of genes involved in lipid metabolism, but also of genes involved in immune response through peroxisome proliferator-activated receptor $\alpha$ $(P P A R \alpha)$-mediated activation.

\section{CONCLUSIONS}

Results of the present study suggest pleiotropic effects of DGAT1 gene polymorphism on the energy metabolism and immune system in the transcriptome of the mammary gland. These results provide a first overview from which new research strategies can be launched to elucidate the underlying molecular mechanisms for DGAT1 gene polymorphism and bovine mammary function.

\section{ACKNOWLEDGMENTS}

The authors gratefully acknowledge the financial support of WUR investment funds (BAS code KB-05-003040-ASG-V-2), and of "Comissionat per a Universitats i Recerca del DIUE" of the Government of Catalonia. The authors are also grateful to Dirk Anjema, Paul Kroon, Johan Meijer, and Peter Jan ten Haken from Wageningen UR Livestock Research (Lelystad, the Netherlands) for surgical biopsies, and to Karel Houwelingen from Wageningen UR Livestock Research and Jordi Estellé from INRA (Dep. Génétique Animale et Biologie Intégrative, Jouy-en-Josas, France) for critical comments.

\section{REFERENCES}

Banos, G., J. A. Woolliams, B. W. Woodward, A. B. Forbes, and M. P. Coffey. 2008. Impact of single nucleotide polymorphisms in leptin, leptin receptor, growth hormone receptor, and diacylglycerol acyltransferase (DGAT1) gene loci on milk production, feed, and body energy traits of UK dairy cows. J. Dairy Sci. 91:3190-3200. 
Berry, D. P., D. Howard, S. O'Boyle, S. Waters, J. F. Kearney, and M. McCabe. 2010. Associations between the K232A polymorphism in the diacylglycerol- $O$-transferase 1 (DGAT1) gene and performance in Irish Holstein-Friesian dairy cattle. Ir. J. Agric. Food Res. 49:1-9.

Bionaz, M., B. J. Thering, and J. J. Loor. 2012. Fine metabolic regulation in ruminants via nutrient-gene interactions: Saturated longchain fatty acids increase expression of genes involved in lipid metabolism and immune response partly through PPAR- $\alpha$ activation. Br. J. Nutr. 107:179-191.

Blum, Y., G. Le Mignon, S. Lagarrigue, and D. Causeur. 2010. A factor model to analyze heterogeneity in gene expression. BMC Bioinformatics 11:368.

Boichard, D., C. Grohs, F. Bourgeois, F. Cerqueira, R. Faugeras, A. Neau, R. Rupp, Y. Amigues, M. Boscher, and H. Levéziel. 2003. Detection of genes influencing economic traits in three French dairy cattle breeds. Genet. Sel. Evol. 35:77-101.

Bouwman, A. C., H. Bovenhuis, M. H. Visker, and J. A. van Arendonk. 2011. Genome-wide association of milk fatty acids in Dutch dairy cattle. BMC Genet. 12:43-55.

Bünger, M., H. M. van den Bosch, J. van der Meijde, S. Kersten, G. J. E. J. Hooiveld, and M. Müller. 2007. Genome-wide analysis of PPAR $\alpha$ activation in murine small intestine. Physiol. Genomics 30:192-204.

Caesar, R., M. Manieri, T. Kelder, M. Boekschoten, C. Evelo, M. Müller, T. Kooistra, S. Cinti, R. Kleemann, and C. A. Drevon. 2010. A combined transcriptomics and lipidomics analysis of subcutaneous, epididymal and mesenteric adipose tissue reveals marked functional differences. PLoS ONE 5:e11525. http://dx.doi.org/10.1371/ journal.pone.0011525.

Causeur, D., C. Friguet, M. Houee-Bigot, and M. Kloareg. 2011. Factor analysis for multiple testing (FAMT): An R package for large-scale significance testing under dependence. J. Stat. Softw. 40:1-19.

Connor, E. E., S. Siferd, T. H. Elsasser, C. M. Evock-Clover, C. P. Van Tassell, T. S. Sonstegard, V. M. Fernandes, and A. V. Capuco. 2008. Effects of increased milking frequency on gene expression in the bovine mammary gland. BMC Genomics 9:362.

Contreras, G. A., and L. M. Sordillo. 2011. Lipid mobilization and inflammatory responses during the transition period of dairy cows. Comp. Immunol. Microbiol. Infect. Dis. 34:281-289.

Danchin-Burge, C., S. J. Hiemstra, and H. Blackburn. 2011. Ex situ conservation of Holstein-Friesian cattle: Comparing the Dutch, French, and US germplasm collections. J. Dairy Sci. 94:41004108.

Donaldson, L., T. Vuocolo, C. Gray, Y. Strandberg, A. Reverter, S. McWilliam, Y. Wang, K. Byrne, and R. Tellam. 2005. Construction and validation of a bovine innate immune microarray. BMC Genomics 6:135.

Farr, V. C., K. Stelwagen, L. R. Cate, A. J. Molenaar, T. B. McFadden, and S. R. Davis. 1996. An improved method for the routine biopsy of bovine mammary tissue. J. Dairy Sci. 79:543-549.

Friguet, C., M. Kloareg, and D. Causeur. 2009. A factor model approach to multiple testing under dependence. J. Am. Stat. Assoc. 104:1406-1415.

Gautier, M., A. Capitan, S. Fritz, A. Eggen, D. Boichard, and T. Druet. 2007. Characterization of the DGAT1 K232A and variable number of tandem repeat polymorphisms in French dairy cattle. J. Dairy Sci. 90:2980-2988.
Grisart, B., F. Farnir, L. Karim, N. Cambisano, J. J. Kim, A. Kvasz, M. Mni, P. Simon, J. M. Frere, W. Coppieters, and M. Georges. 2004. Genetic and functional confirmation of the causality of the DGAT1 K232A quantitative trait nucleotide in affecting milk yield and composition. Proc. Natl. Acad. Sci. USA 101:2398-2403.

Koliwad, S. K., R. S. Streeper, M. Monetti, I. Cornelissen, L. Chan, K. Terayama, S. Naylor, M. Rao, B. Hubbard, and R. V. Farese Jr. 2010. DGAT1-dependent triacylglycerol storage by macrophages protects mice from diet-induced insulin resistance and inflammation. J. Clin. Invest. 120:756-767.

Lê, S., J. Josse, and F. Husson. 2008. FactoMineR: An R package for multivariate analysis. J. Stat. Softw. 25:1-18.

Loor, J. J., H. M. Dann, R. E. Everts, R. Oliveira, C. A. Green, N. A. Guretzky, S. L. Rodriguez-Zas, H. A. Lewin, and J. K. Drackley. 2005. Temporal gene expression profiling of liver from periparturient dairy cows reveals complex adaptive mechanisms in hepatic function. Physiol. Genomics 23:217-226.

Loor, J. J., H. M. Dann, N. A. Guretzky, R. E. Everts, R. Oliveira, C. A. Green, N. B. Litherland, S. L. Rodriguez-Zas, H. A. Lewin, and J. K. Drackley. 2006. Plane of nutrition prepartum alters hepatic gene expression and function in dairy cows as assessed by longitudinal transcript and metabolic profiling. Physiol. Genomics 27:29-41.

Mach, N., A. A. A. Jacobs, L. Kruijt, J. van Baal, and M. A. Smits. 2011. Alteration of gene expression in mammary gland tissue of dairy cows in response to dietary unsaturated fatty acids. Animal 5:1217-1230.

McCudden, C. R., M. D. Hains, R. J. Kimple, D. P. Siderovski, and F. S. Willard. 2005. G-protein signaling: Back to the future. Cell. Mol. Life Sci. 62:551-577.

Odong, T. L., J. van Heerwaarden, J. H. Jansen, T. J. L. van Hintum, and F. A. van Eeuwijk. 2011. Determination of genetic structure of germplasm collections: Are traditional hierarchical clustering methods appropriate for molecular marker data? Theor. Appl. Genet. 123:195-205.

Ogorevc, J., T. Kunej, A. Razpet, and P. Dovc. 2009. Database of cattle candidate genes and genetic markers for milk production and mastitis. Anim. Genet. 40:832-851.

Schennink, A., J. M. L. Heck, H. Bovenhuis, M. H. P. W. Visker, H. J. F. van Valenberg, and J. A. M. van Arendonk. 2008. Milk fatty acid unsaturation: Genetic parameters and effects of stearoyl-CoA desaturase $(S C D 1)$ and acyl CoA: Diacylglycerol acyltransferase 1 (DGAT1). J. Dairy Sci. 91:2135-2143.

Schennink, A., W. M. Stoop, M. H. P. W. Visker, J. M. L. Heck, H. Bovenhuis, J. J. van der Poel, H. J. F. van Valenberg, and J. A M. van Arendonk. 2007. DGAT1 underlies large genetic variation in milk-fat composition of dairy cows. Anim. Genet. 38:467-473.

Subramanian, A., P. Tamayo, V. K. Mootha, S. Mukherjee, B. L. Ebert, M. A. Gillette, A. Paulovich, S. L. Pomeroy, T. R. Golub, E. S. Lander, and J. P. Mesirov. 2005. Gene set enrichment analysis: A knowledge-based approach for interpreting genome-wide expression profiles. Proc. Natl. Acad. Sci. USA 102:15545-15550.

Thaller, G., W. Kramer, A. Winter, B. Kaupe, G. Erhardt, and R. Fries. 2003. Effects of DGAT1 variants on milk production traits in German cattle breeds. J. Anim. Sci. 81:1911-1918. 